\title{
Influence of phonon dispersion on exciton damping in ionic crystals
}

\author{
N.I. Grigorchuk \\ Bogolyubov Institute for Theoretical Physics, NAS of Ukraine, \\ e-mail:ngrigor@nolin.bitp.kiev.ua
}

\begin{abstract}
The manifestation of phonon dispersion on frequency and temperature dependence of exciton damping is investigated theoretically for the models of crystal with the large and small exciton radii. The correlation between the dispersion and the frequency intervals with phonon absorption and emission is discussed for the exciton of $\mathrm{TlBr}, \mathrm{ZnS}$ and some other crystals as examples. It is demonstrated that depending on frequency the dispersion results both in increase and reduction of the exciton damping. In addition, the intensity of exciton damping close to the peaks of phonon absorption and emission is varied. The change of the tendency of influence of dispersion on damping for some fixed frequency with temperature growing is noticed.
\end{abstract}

Keywords: exciton damping, phonon dispersion, absorption, emission, crystal.

Paper received 31.10.00; revised manuscript received 11.10.01; accepted for publication 12.12.01.

\section{Introduction}

The phonons cause power losses for excitations [1] and assist appreciable influence on the physical properties of materials [2]. The scattering of excitons by phonons is recently treated more intensively for both low-dimensional [3] and anisotropic [4] crystals. In these materials, the energy of exciton coupling grows in comparison with its volumetric meaning [5] and the power exchange with phonons becomes more essential [6,7]. The detailed study of the phonon dispersion is important both owing to the practical application in device making and for the deeper understanding of the nature of microprocesses in solid state.

The relaxation of the energy of an exciton goes the most effectively through its interaction with an optical phonon [8] (except for low temperatures, at which the acoustic phonons can give the comparable contribution). Among other branches of vibrations, one allocate the interaction with longitudinal optical (LO) phonons [9]. As a most energetic, these phonons have a significant dispersion for majority of directions of the wave vector [10]. However, the LO-phonon dispersion has been frequently neglected supposing merely that all oscillators have identical frequency (the well-known Einstein model). Such approach can result in loss of an important information. For instance, the investigation of the polaron mobility in one-dimensional conductors [11] has shown the essential influence of the phonon dispersion. In three-dimensional crystals [12], it was established that the phonon dispersion appreciably changes a low frequency asymptotic of conductivity. In work [13], for strongly coupled electronphonon system, it was revealed that the dispersion of phonon frequency can cause washing out the oscillations in angle-resolved photoemission spectra.

The principal objective of this work is to study the influence of the LO-phonons dispersion on frequency and temperature dependence of exciton damping within the limits of weak coupling of excitons with phonons and one-phonon approximation.

Earlier this effect was studied in detail by us using cosine law for dispersion [14]. Owing to the peculiarities of the edges of the Brillouin zone, there is a great interest to study the damping for other laws of phonon dispersion as well.

For the illustration, we use the model of $\mathrm{TlBr}$ and $\mathrm{ZnS}$ crystals with large and small exciton radii, respectively. Further, the paper is constructed as follows. In the second section the basic formulas for calculations are given. In the third section the obtained results are discussed and in the final fourth section, the conclusions are presented. 


\section{N.I. Grigorchuk: Influence of phonon dispersion on exciton damping in ionic crystals}

\section{Basic formulae of a task}

Let's treat the cubic crystal as the system of periodically placed quantum oscillators, in which under action of an external excitation the excitons can be create. It is supposed that the exciton realizing coherent motion in a phonons environment and can be described by the Bloch wave. Then, in the case of a weak exciton-phonon coupling the one-phonon scattering processes play the most important role. Exciton collisions with phonons results in restriction of its lifetime and to the damping $\Gamma$ of an exciton wave that in quantum language can be described as the sum of processes with the absorption $\Gamma^{+}$and emission $\Gamma^{-}$of phonons:

$$
\Gamma=\Gamma^{+}+\Gamma^{-} \text {. }
$$

In the first Born approximation the damping depending on frequency of the incident wave $\omega$ and temperature $\mathrm{T}$ of the crystal can be presented in a general form as [15]:

$$
\begin{aligned}
& \Gamma^{ \pm}(\mathbf{k}, \omega, T)=2 \pi \sum_{\mathbf{q}}\left|G_{j O}(\mathbf{q})\right|^{2}\left(N_{\mathbf{q}}(T)+\frac{1}{2} \mp \frac{1}{2}\right) \times \\
& \times \delta\left[\hbar \omega-E(\mathbf{k} \pm \mathbf{q}) \pm \Omega_{j O}(\mathbf{q})\right]
\end{aligned}
$$

In this formula $G_{j O}(\mathbf{q})$ is the coupling function of excitons with the longitudinal $(j=L)$ or transverse $(j=T)$ optical phonons, $E(\mathbf{k})$ and $\Omega_{j O}(\mathbf{q})$ are, accordingly, the energy of an exciton and $j O$-phonon in the crystal, $\delta[\ldots]$ is the Dirac delta-function, $N_{\mathbf{q}}(T)$ is the average number of phonons with a quasi-momentum $\mathbf{q}$ which obey to the Bose statistics, $\mathbf{k}$ is the exciton quasi-momentum. Longitudinal and transverse modes of the optical vibrations generally are the solutions of the macroscopic equation of motions for relative displacement $\mathbf{U}$ of ions connected to the appropriate Maxwell equations, namely

$$
\left\{\begin{array}{c}
\rho \ddot{\mathbf{U}}=-\rho \omega_{T O}^{2} \mathbf{U}+e_{i} \mathbf{E}+c_{11} \nabla(\nabla \mathbf{U})+c_{44} \nabla \times \nabla \times \mathbf{U}, \\
\nabla(\varepsilon \mathbf{U})=0, \\
\ddot{\mathbf{E}}_{T}=\frac{c^{2}}{\varepsilon \mu_{0}} \nabla^{2} \mathbf{E}_{T} .
\end{array}\right.
$$

Table 1. Basic properties of optical modes
Here $\rho$ is the reduced mass density of a material, $e_{i}$ is the effective ionic charge density, $\mathbf{E}$ is the macroscopic electrical field, $\omega_{T O}$ is the frequency of TO-mode, $c_{11}$ and $c_{44}$ are optical-mode elastic constants, $\mathbf{E}_{T}$ is any transversely polarized component of the electrical field, $\mu_{0}$ is the magnetic permeability of free space. The wellknown properties of these modes (for example, [16]) are given in Table 1.

Taking into account the expressions for the energy of optical modes given in this Table we shall calculate the damping of an exciton in the crystal. Let's choose in Eq.(2) the dependence of exciton energy on quasi-momenta of exciton and phonon as

$E(\mathbf{k} \pm \mathbf{q})=E_{0}+\frac{\hbar^{2}}{2 M}(\mathbf{k} \pm \mathbf{q})^{2}, \quad M=m_{e}+m_{h}$,

where $E_{0}$ is the exciton band bottom energy, $m_{e}$ is the electron mass, $m_{h}$ is the hole mass. Let's substitute both the Eq. (4) and one of expressions of Table 1 in the formula (2). We shall convert summation over $\mathbf{q}$ in Eq. (2) to the appropriate integration. The integration will be performed in the spherical system of coordinates. Integral over polar corner gives $2 \pi$, and the integration over azimuthal corner is easy for executing by using the $\delta$ functions. Then, one obtains

$$
\begin{aligned}
& \Gamma^{ \pm}(k, \omega, T)=\frac{M V}{2 \pi \hbar^{2} k}\left|1 \pm \frac{d_{j} / n}{\sqrt{1-d_{j}\left(q_{ \pm} / q_{0}\right)}}\right|^{-1} \times \\
& \times \int_{-k+q_{ \pm}}^{k+q_{ \pm}} d q q G_{j O}^{2}(q)\left[N_{\mathbf{q}}(T)+\frac{1}{2} \mp \frac{1}{2}\right]
\end{aligned}
$$

where

$$
\begin{aligned}
& \left(\frac{q_{ \pm}}{q_{0}}\right)^{2} \cong \frac{2}{n}\left(\frac{\hbar \omega-E_{0}}{\Omega_{j O}}-\frac{d_{j}}{n}\right) \pm \\
& \pm \sqrt{1+\left(\frac{d_{j}}{n}\right)^{2}+d\left(\frac{k}{q_{0}}\right)^{2}-\frac{\hbar \omega-E_{0}}{\Omega_{j O}}}, \\
& q_{0}^{2}=n \frac{M \Omega_{j O}}{\hbar^{2}}, \quad d_{j}=n \frac{M v_{j}^{2}}{\Omega_{j O}}, \quad j=L, T,
\end{aligned}
$$

\begin{tabular}{l|l|c|c}
\hline \hline Mode & Dispersion & Electrical field & Permeability \\
\hline LO & $\Omega^{2}(q)=\Omega_{L O}^{2}-\hbar^{2} q^{2} v_{L}^{2}$ & $\mathbf{E}_{L}=-e_{i} / \varepsilon_{\infty} \mathbf{U}_{L}$ & $\varepsilon(\omega)=0$ \\
\hline TO & $\Omega^{2}(q)=\Omega_{T O}^{2}-\hbar^{2} q^{2} v_{T}^{2}$ & $\mathbf{E}_{T}=0$ & $\varepsilon(\omega) \rightarrow \infty$ \\
\hline \hline
\end{tabular}

$\Omega_{L O}^{2}=\left(\varepsilon_{0} / \varepsilon_{\infty}\right) \Omega_{T O}^{2}, \quad e_{i}^{2}=\varepsilon_{\infty} \rho\left(\Omega_{L O}^{2}-\Omega_{T O}^{2}\right) / \hbar^{2}$,

$v_{L}^{2}=c_{11} / \rho, \quad v_{T}^{2}=c_{44} / \rho$. 


\section{N.I. Grigorchuk: Influence of phonon dispersion on exciton damping in ionic crystals}

$V$ is the volume of the crystal, $n=1,2,3, \ldots$ are some integers (for maximal $n$ the value of $d_{j}$ ought to be less than unity). The values $q_{ \pm}$con be obtained from algebtic equation of the fourth order. Their exact meaning is rather cumbersome, thereofore, we have kept only the essential terms in Eq.(6)The limits of integration in Eq. (5) should satisfy the conditions:

$\left\{\begin{array}{c}k+q_{ \pm} \leq q_{\max } \\ -k+q_{ \pm} \geq 0\end{array}\right.$

where $q_{\max }=\left(6 \pi^{2} / V_{0}\right)^{1 / 3}, V_{0}$ is the volume of an elementary cell. Besides, from Eq.(6) follows that

$\hbar \omega-E_{0} \leq \Omega_{j O}\left(\frac{n}{2 d_{j}}+\frac{d_{j}}{2 n}+\frac{n}{2}\left(\frac{k}{q_{0}}\right)^{2}\right), \quad d_{j} \neq 0$.

Above entered parameter $d_{j}$ can be considered as parameter describing the dispersiveness of the $j$-th mode of optical vibrations in the polar crystal.

The further calculation of Eq. (5) in an analytical form is possible for elementary kind of the coupling function $G_{j O}(q)$, without any restrictions on the value of an exciton quasi-momentum $k$, or for a general view of the function $G$ with $k \rightarrow 0$. First, let us consider the former case.

We shall be limited hereinafter to the interaction of excitons only with the longitudinal optical (LO) phonons. Let the length of a phonon wave $\lambda_{p h}$ be much more than, the radius of an exciton $r_{e x}$. Then, the coupling function of excitons with LO-phonons (having the dispersion $d_{L}$ ) is taken to be

$$
\begin{aligned}
& G_{L O}(q)=2 \Omega_{0}\left(\alpha_{h}^{2}-\alpha_{e}^{2}\right) \times \\
& \times q \sqrt{\frac{4 \pi}{V} \alpha R}\left[1-d_{L}\left(\frac{q}{q_{0}}\right)^{2}\right]^{1 / 4} .
\end{aligned}
$$

Here, $\Omega_{0}$ is the energy of optical phonon with zero momentum,

$$
\alpha=\frac{e^{2}}{\hbar} \sqrt{\frac{M}{2 \Omega_{0}}}\left(\frac{1}{\varepsilon_{\infty}}-\frac{1}{\varepsilon_{0}}\right), \quad R=\sqrt{\frac{\hbar^{2}}{2 M \Omega_{0}}}
$$

are, accordingly, the polaronic coupling constant and the radius of polaron with mass $M, \varepsilon_{\infty}$ and $\varepsilon_{0}$ are the dielectric constants for high and low frequencies, respectively,

$\alpha_{i}=\frac{r_{e x}}{2} \frac{m_{i}}{M}, \quad i=e, h$

The integration in Eq. (5) with the account for Eq.(10) gives

$$
\begin{aligned}
& \Gamma^{ \pm}(k, \omega, T) \approx 16 \alpha R \Omega_{0}\left(\alpha_{h}^{2}-\alpha_{e}^{2}\right)^{2} q_{0}^{2} q_{ \pm} \times \\
& \times\left(k^{2}+q_{ \pm}^{2}\right) \frac{1-d_{L}\left(\frac{k^{2}+q_{ \pm}^{2}}{q_{0}^{2}}\right)}{\mid \frac{d_{l}}{n} \pm \sqrt{1-d_{L}\left(\frac{k^{2}+q_{ \pm}^{2}}{q_{0}^{2}}\right) \mid} \times} \\
& \times\left\{\frac{1}{\exp \left[\frac{\Omega_{0}}{k_{B} T} \sqrt{\left.1-d_{L}\left(\frac{k^{2}+q_{ \pm}^{2}}{q_{0}^{2}}\right)\right]}-1\right.}+\frac{1}{2} \mp \frac{1}{2}\right\},
\end{aligned}
$$

where quasi-momentums $k$ and $q_{ \pm}$satisfy the conditions of Eq. (8) and also to the criterion:

$k^{2}+q_{ \pm}^{2} \leq q_{0}^{2} / d_{L}$.

The dependence $\Gamma^{ \pm}$on the frequency is set only through the parameter $q_{ \pm}$of Eq. (6). We have also used the identity

$$
\begin{aligned}
& 1-d_{j}\left(\frac{q_{ \pm}}{q_{0}}\right)^{2} \equiv \\
& \equiv\left(\sqrt{\left.1+\left(\frac{d_{j}}{n}\right)^{2}+d_{j}\left(\frac{k}{q_{0}}\right)^{2}-\frac{2 d_{j}}{n} \frac{\hbar \omega-E_{0}}{\Omega_{j O}} \mp \frac{d_{j}}{n}\right)^{2},}\right.
\end{aligned}
$$

with $j=L$ at calculation of Eq. (13).

Formula (13) describes the damping of an exciton by absorption or emission of a phonon moving under some corner $\theta$ with respect to the direction of exciton movement k. Equation (13), in other words, determines the probability that an exciton with a wave vector $\mathbf{k}$ can absorb or emit a phonon with the quasi-momentum $q_{+}$and dispersion $d_{L}$.

If the effective mass of an electron and hole are coincides, the scattering of an excitons by LO-phonons does not occur. There comes the mutual compensation of the polarization in accordance to the model of independent polarization of the lattice by an electron and hole taken separately, chosen by us in Eq. (10).

Let's consider now the case of the small exciton quasimomentums. In this case, in Eq. (5) there arises uncertainty relation of the type $0 / 0$, opening which, we arrive at the following expression

$$
\begin{aligned}
& \Gamma^{ \pm}(k \rightarrow 0, \omega, T)=\frac{V}{n \pi \Omega_{0}} q_{0}^{2} q_{ \pm} \times \\
& \left.\times \frac{G_{j O}^{2}\left(q_{ \pm}\right)}{1 \pm \frac{d_{j} / n}{\sqrt{1-d_{j}\left(q_{ \pm} / q_{0}\right)^{2}}}} \mid N\left(q_{ \pm}, T\right)+\frac{1}{2} \mp \frac{1}{2}\right] .
\end{aligned}
$$




\section{N.I. Grigorchuk: Influence of phonon dispersion on exciton damping in ionic crystals}

Within the framework of one-phonon approximation, the function $G$ here can be arbitrary function of $q_{ \pm}$. Here the conditions (8) are found to be

$$
\begin{aligned}
& -\Omega_{j O} \leq \hbar \omega-E_{0} \leq \\
& \leq \Omega_{j O}\left[\frac{n}{2}\left(\frac{q_{\text {max }}}{q_{0}}\right)^{2}-\sqrt{1-d_{j}\left(\frac{q_{\text {max }}}{q_{0}}\right)^{2}}\right],
\end{aligned}
$$

for processes with the phonon absorption $\Gamma^{+}$, and

$$
\begin{aligned}
& \Omega_{j O} \leq \hbar \omega-E_{0} \leq \\
& \leq \Omega_{j O}\left[\frac{n}{2}\left(\frac{q_{\max }}{q_{0}}\right)^{2}+\sqrt{1-d_{j}\left(\frac{q_{\max }}{q_{0}}\right)^{2}}\right],
\end{aligned}
$$

for processes with the phonon emission $\Gamma^{-}$. From Eqs (17), (18) follows, that maximal dispersion in the polar crystals may achieve the magnitude:

$$
d_{\max }=\left(\frac{q_{0}}{q_{\max }}\right)^{2},
$$

Otherwise, this substantially means that the maximal pulse of a phonon in the crystal should satisfy also the criterion: $q_{\max } \leq \omega_{j O} / v_{j}$ (where $\left.\omega_{j O}=\hbar^{-1} \Omega_{j O}\right)$.

Let's choose $G$ as linear in operator of exciton-phonon interaction coupling function for 1 s exciton:

$$
\begin{aligned}
& G_{L O}(q)=\sqrt{\frac{4 \pi}{V} \alpha R \Omega_{0}} \frac{\sqrt{\Omega_{L O}(q)}}{q} \times \\
& \times\left[\left(1+\alpha_{e}^{2} q^{2}\right)^{-2}-\left(1+\alpha_{h}^{2} q^{2}\right)^{-2}\right] .
\end{aligned}
$$

Then, the damping of an exciton on the longitudinal vibrations of the lattice in accordance with Eq. (16) is determined as

$$
\begin{aligned}
& \Gamma^{ \pm}(0, \omega, T)=2 \sqrt{\frac{2}{n}} \alpha \Omega_{0} \frac{1-d_{L}\left(\frac{q_{ \pm}}{q_{0}}\right)^{2}}{\left[\frac{q_{ \pm}}{q_{0}}\left(\frac{d_{L}}{n} \pm \sqrt{1-d_{L}\left(\frac{q_{ \pm}}{q_{0}}\right)^{2}}\right)\right.} \times \\
& \times\left[\frac{1}{\left[1+\beta_{e}^{2}\left(\frac{q_{ \pm}}{q_{0}}\right)^{2}\right]^{2}}-\frac{1}{\left[1+\beta_{h}^{2}\left(\frac{q_{ \pm}}{q_{0}}\right)^{2}\right]^{2}}\right]^{2} \times
\end{aligned}
$$

$$
\times\left\{\frac{1}{\exp \left[\frac{\Omega_{0}}{k_{B} T} \sqrt{1-d_{L}\left(\frac{q_{ \pm}}{q_{0}}\right)^{2}}\right]-1}+\frac{1}{2} \mp \frac{1}{2}\right\},
$$

where $\beta_{i}=\alpha_{i} q_{0}$. Notice that the formula (21) describes the damping not only close to the bottom of an exciton zone, but on distances of the order of an energy characteristic to the dispersionless phonon as well. It follows from the dependence of exciton energy in Eq. (2) on the phonon quasi-momentum.

We put $q_{0}=q_{\text {max }}$ for one-phonon approach. From conditions, ensuring the frequencies lacing for phonon absorption or emission (in absence of its dispersion) it is possible to determine the parameter $n$. Thus, we find that at $n=4$ the conditions (17), (18) for Eq. (21) are rewritten as:

$-\Omega_{0} \leq \hbar \omega-E_{0} \leq \Omega_{0}\left(2-\sqrt{1-d_{L}}\right)$,

for $\Gamma^{+}$, and

$\Omega_{0} \leq \hbar \omega-E_{0} \leq \Omega_{0}\left(2+\sqrt{1-d_{L}}\right)$,

for $\Gamma^{-}$. Therefore, formally

$0 \leq d_{L} \leq 1$

Though for concrete crystal the value of $d$ is quite certain for any given mode $j$, but studying the effect of phonon dispersion on exciton damping in the crystal, was allowed to vary it in the limits of (24). As it is seen from Eqs (22), (23), at maximal dispersion $(d=1)$ the range with the phonon emission is narrowed by the value of the energy LO-phonon while the range with phonon absorption extends by the same quantity of energy. The magnitude $d$ determines a share of the contribution of the phonon zone to the exciton scattering.

In the case when phonon dispersion is neglected $(d=0)$ the expression (8) with the account for Eq. (9) can be reduced to the known from earlier works (see, for example, [17]).

As models for calculations the number of ionic crystals were chosen with parameters given in Table 2 . The exciton radius was calculated here by the formula $r_{e x}=$ $=(\hbar / e)^{2} \varepsilon_{0} / \mu, 1 / \mu=1 / m_{e}+1 / m_{h}$. It is necessary to notice that the problem of determination of exciton radius is connected with the problem of a choice of dielectric constant $\varepsilon$ [23]. If the angular frequency of an exciton $\omega_{e x}=\hbar /\left(\mu r_{e x}^{2}\right)$ is less or does not exceed frequency of a longitudinal mode of optical vibrations $\Omega_{0}$, the displacement of vibrations of a lattice follows to the exciton motion. Then the lattice is polarized, and, for Coulomb interaction it is necessary to choose the low-frequency di- 


\section{N.I. Grigorchuk: Influence of phonon dispersion on exciton damping in ionic crystals}

electric constant $\varepsilon_{0}$. On the contrary in the case, when the angular frequency of an exciton is greater than $\Omega_{0}$, than the exciton follows to the lattice motion, and the choice of high-frequency dielectric constant $\varepsilon_{\infty}$ is more acceptable. For the crystals given in Table 2, the constant $\varepsilon_{0}$ proved to be proper.

\section{Discussion of results}

For illustration we have chosen from Table $2 \mathrm{TlBr}$ and $\mathrm{ZnS}$ (sphalerit) crystals having most contrast parameters $\varepsilon_{0}, r_{e x}, \Omega_{0}$. In figures 1 and 2 , the frequency dependence of excitons damping are given in the presence (curves 1) or in the absence (curves 2) of dispersions of optical vibrations of the lattice. The calculations are performed for room temperature $(T=300 \mathrm{~K})$, at which effect of dis-

Table 2. Parameters of ionic crystals persion are the most distinct. As seen from the figures, the frequency dependence of damping represents a doublehumped curve, according to processes involving absorption and emission LO-phonon in the crystal.

The scattering of an exciton begins with the energy $E_{0}-\Omega_{0}$. The intensity of damping grows quickly with increase of difference in effective masses of an electron and hole. This growth is restrained by reduction of the electron-phonon coupling with increasing the wave vector $\mathbf{q}$, when the phonon wavelength becomes less than the exciton radius. Especially remarkable such reduction is for crystals with large exciton radii (as considered $\mathrm{TlBr}$ ) and poorly manifest itself for excitons with small exciton radii (as in $\mathrm{ZnS}$ ) for which the maximum of exciton-phonon coupling is displaced to the side of shorter waves or even may fall outside the frequency limits of one-phonon interval. The competition of these factors forms the maximums in damping.

\begin{tabular}{lccccccccc}
\hline \hline Crystals & $\varepsilon_{0}$ & $\varepsilon_{\infty}$ & $m_{e} / m_{0}$ & $m_{h} / m_{0}$ & $\Omega_{0,} \mathrm{~cm}^{-1}$ & $r_{e x}, \mathrm{~A}^{\circ}$ & $a, \mathrm{~A}^{\circ}$ & $\alpha$ & $\mathrm{C}_{11} \cdot 10^{11},(\mathrm{dyn} . / \mathrm{cm})^{2}$ \\
\hline $\mathrm{MgO}^{*}$ & 9.8 & 2.97 & 0.25 & 3.20 & 726 & 22.4 & 4.21 & 5.36 & 29.6 \\
\hline $\mathrm{TlBr}^{* *}$ & 35.1 & 5.4 & 0.18 & 0.38 & 115 & 152.1 & 3.97 & 3.62 & 3.78 \\
\hline $\mathrm{TlCl}^{* *}$ & 37.6 & 5.1 & 0.37 & 0.36 & 173 & 109 & 3.84 & 3.65 & 4.04 \\
\hline $\mathrm{GaP}^{* *}$ & 11.0 & 9.1 & 0.17 & 0.24 & 403 & 59.0 & 5.45 & 0.2 & 14.5 \\
\hline $\mathrm{Cu}_{2} \mathrm{O}^{* * *}$ & 7.1 & 6.2 & 0.61 & 0.84 & 606 & 10.63 & 2.46 & 0.33 & 11.6 \\
\hline $\mathrm{ZnS}^{* * * *}$ & 8.6 & 5.2 & 0.25 & 0.59 & 352 & 25.9 & 5.41 & 1.23 & 10.5 \\
\hline $\mathrm{AgBr}^{* * *}$ & 10.6 & 4.68 & 0.22 & 0.70 & 140 & 33.4 & 5.77 & 3.21 & 5.63 \\
\hline \hline
\end{tabular}

The note. * On the data [18]. ** On the data [19]. *** On the data [20]. **** On the data [21]. The meaning of constants $\mathrm{c}_{11}$ are given on the data [22] at room temperature.

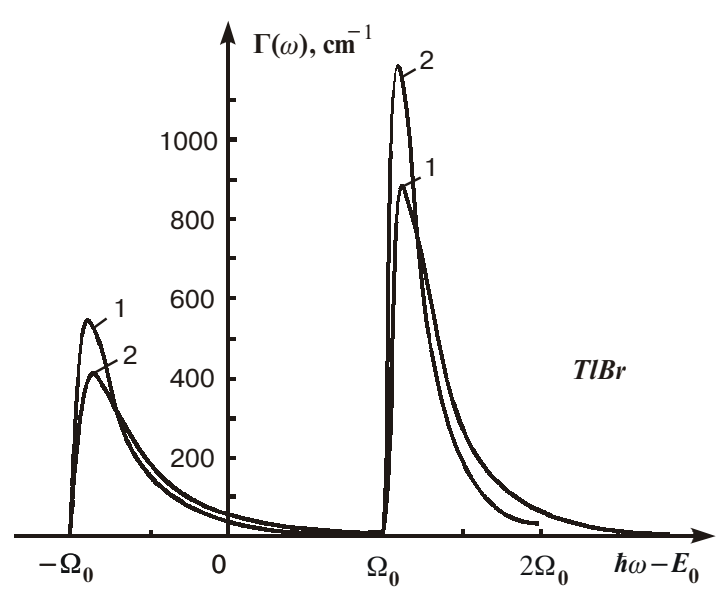

Fig .1. Frequency dependence of exciton damping for $\mathrm{TlBr}$ crystal at room temperature for different values of LO-phonon dispersion: $d=0$ (curve 1$), d=1$ (curve 2).

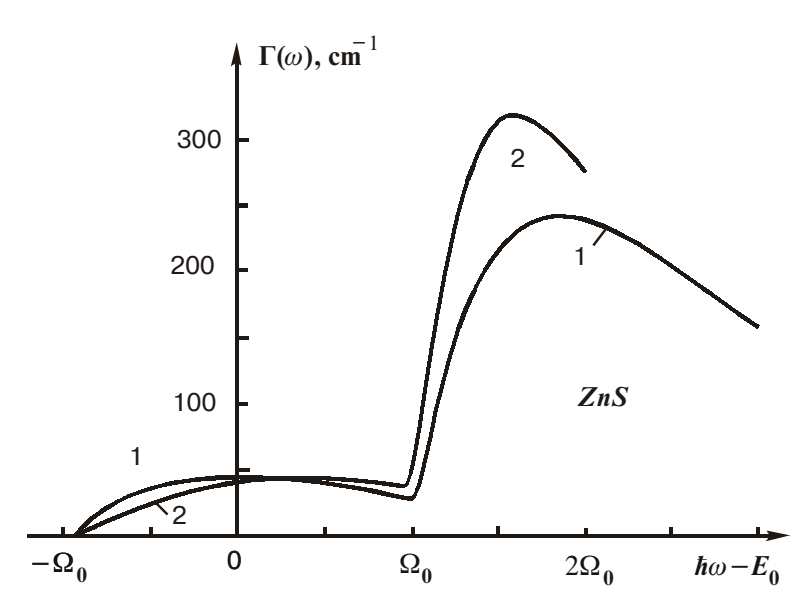

Fig. 2. Frequency dependence of exciton damping for $\mathrm{ZnS}$ crystal at room temperature for different values of LO-phonon dispersion: $d=0$ (curve 1$), d=1$ (curve 2 ). 


\section{N.I. Grigorchuk: Influence of phonon dispersion on exciton damping in ionic crystals}

Starting with the energy $E_{0}+\Omega_{0}$ alongside with absorption the processes with phonon emission begin to give contribution to the damping as well, which is sustained down to helium temperatures inclusively. The summation of all named processes for this spectrum range causes the higher intensity of damping.

Increase in phonon band dispersion results in the weak displacement of the maximum of damping (below $E_{0}$ ) to the short-wave side of the spectrum. This displacement is due to the fact that with increasing phonon wave vector the LO-phonons frequency decreases, and contribution of phonons with identical quasi-momentums is displaced to the side of shorter waves. On the other hand, with dispersion growth the processes with phonon emission come to the end at lesser frequencies (see (23)), which results in displacement of the second maximum (above $E_{0}$ ) to the long-wave side of the spectrum. As seen from Figs 1, 2, the growth of dispersion determines the downturn of a damping maximum in the range with phonon absorption and its increasing in the range with phonon emission. Such behavior follows from restrictions (22), (23) to the frequency range of variation of $\Gamma^{+}$and $\Gamma^{-}$.

The displacement of damping maxima (both higher and lower $E_{0}$ ) are more considerably for crystals with small exciton radii $\left(\mathrm{Cu}_{2} \mathrm{O}, \mathrm{AgBr}\right.$ and $\mathrm{ZnS}$ (Fig. 2)), whereas the change of intensities of damping is more essential for crystals with the large exciton radii (as $\mathrm{TlCl}$ ). For the former, as a rule, the maximum is pronounced expressed and retained with displacement. This behavior may be considered as a consequence of weakening the excitonphonon coupling in the field of frequencies, where the length of the phonon wave exceeds the exciton radius.

The absolute value of damping of an exciton for the certain crystal, except for told, is determined also by both polaronic coupling constant $\alpha$ and the energy of dispersionless phonon $\Omega_{0}$.

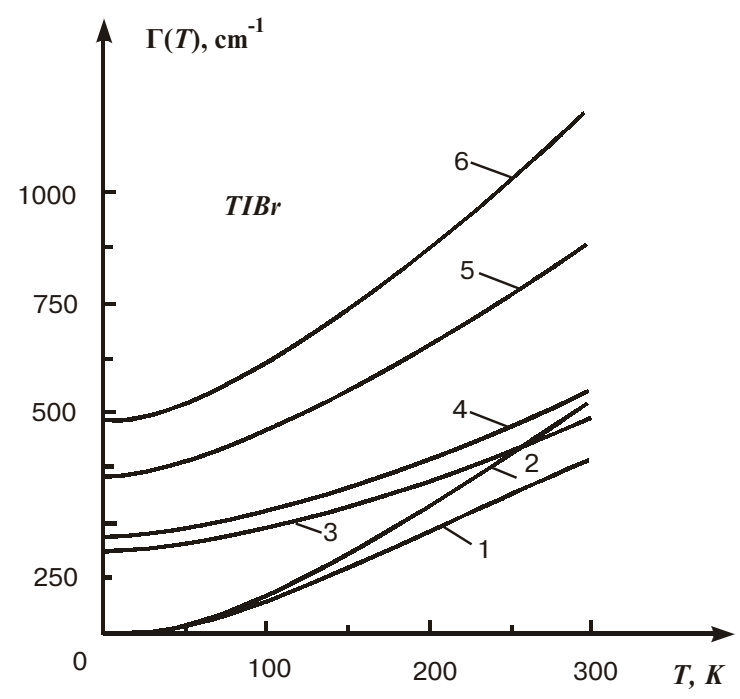

Fig. 3. Temperature dependence of damping for a $\mathrm{TlBr}$ crystal at energies $\hbar \omega-E_{0}=-0.9 \Omega_{0}$ (curves 1,2$),-1.3 \Omega_{0}$ (curves 3, 4), $1.1 \Omega_{0}$ (curves 5,6 ). The curves $1,3,6$ correspond to the presence of dispersion, and its absence is displayed by curves $2,4,5$.
Thus, analyzing the frequency dependence of damping for crystals with considerably distinguished constants, one finds the same tendency, namely, with increasing of phonon dispersion the short-wave wing LO-phonon damping is displaced towards the direction of exciton band bottom. This results in narrowing the frequency interval with phonon emission, and by that, to increase of absolute magnitude of damping. At the same time, the range with phonon absorption extends, accordingly, the intensity of damping falls, especially for crystals with the large exciton radii.

In Figs 3, 4, the results of calculation of damping versus temperature for crystals $\mathrm{TlBr}$ and $\mathrm{ZnS}$ are shown. The calculations were fulfilled for some fixed frequencies in presence (curves 1, 3,6) and in absence (curves 2, 4,6) of phonon dispersion. As a rule, those frequencies got out, for which the display of dispersion was the most appreciable or any other characteristic tendencies were noticed.

So, for all crystals, in the frequency region $\omega \leq$ $\leq\left(E_{0}+\Omega_{0}\right) \hbar^{-1}$, we found that $\Gamma \rightarrow 0$ at $T \rightarrow 0$. That corresponds to usual theoretical conceptions $[9,17]$ and is caused by absence both absorption and emission of phonons in this frequency region.

Depending on the frequency interval, the growth of phonon dispersion can either increase or decrease the exciton damping. In the vicinity of the maximum of phonon absorption, at energies $\hbar \omega-E_{0}=-0.9 \Omega_{0}$ for $\mathrm{TlBr}$ and $\hbar \omega-E_{0}=-0.5 \Omega_{0}$ for $\mathrm{ZnS}$, the dispersion results in reduction of damping (compare curves 1 and 2). On the wing of absorption beginning with the energy $\hbar \omega-E_{0} \leq-0.7 \Omega_{0}$ for $\mathrm{TlBr}$ and $\hbar \omega-E_{0} \leq 0.2 \Omega_{0}$ for $\mathrm{ZnS}$, on the contrary, the dispersion causes growth of the damping.

Such tendency is also developed in the vicinity of the phonon emission maximum (curves 5, 6, Figs 3, 4). It is especially appreciable at energies $\hbar \omega-E_{0}=1.1 \Omega_{0}$ for

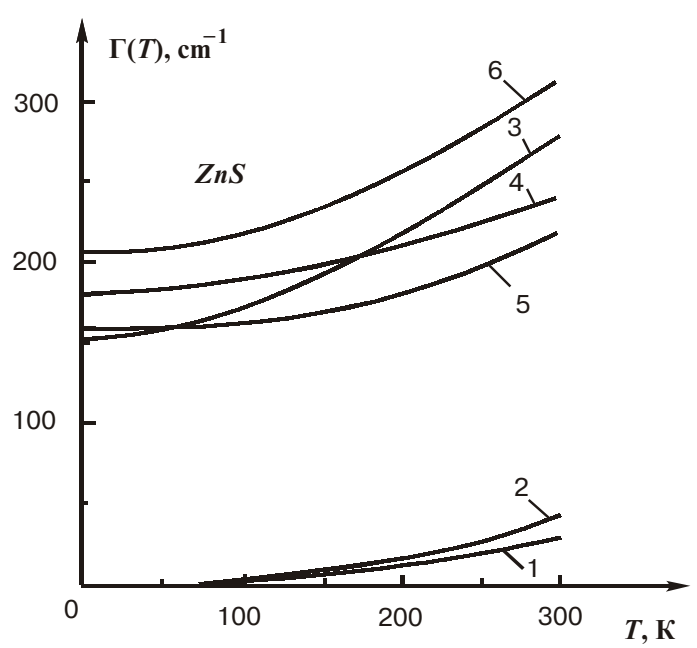

Fig. 4. Temperature dependence of damping for $\mathrm{ZnS}$ crystal at energies $\hbar \omega-E_{0}=-0.5 \Omega_{0}$ (curves 1,2$), 2.0 \Omega_{0}$ (curves 3,4$) 1.5 \Omega_{0}$ (curves 5,6 ). The presence of dispersion is reflected by curves $1,3,6$, and its absence - by curves $2,4,5$. 


\section{N.I. Grigorchuk: Influence of phonon dispersion on exciton damping in ionic crystals}

$\mathrm{TlBr}$ and $\hbar \omega-E_{0}=1.5 \Omega_{0}$ for $\mathrm{ZnS}$, at which the contribution from a phonon dispersion is the greatest (Figs 1, 2). Simultaneously, on the wing with phonon emission the growth of a dispersion again stimulates reduction of exciton damping (curves 3, 4). For TlBr crystals, it occurs at frequencies $\omega \leq\left(E_{0}+1.3 \Omega_{0}\right) \hbar^{-1}$ and for $\mathrm{ZnS}$ at $\omega \leq 2 \Omega_{0} \hbar^{-1}$.

As the rule, the damping of an exciton in the crystal gradually grows with temperature. This growth is approximately identical for most frequencies from onephonon interval as in presence of a dispersion, so in its absence. This tendency is kept for crystals with the large exciton radii. However, for crystals with small exciton radii the interesting phenomena is found out: if in presence of phonon dispersion the exciton damping is small at low temperatures, then with temperature growth, beginning with some critical temperature $T_{c r}$, it starts even to exceed the damping in the absence of a dispersion (see curve 3 in Fig. 4). For $\mathrm{ZnS}$ crystal $T_{c r}=170 \mathrm{~K}$. This effect is due to the fact that with increasing temperature the exciton scattering on the dispersionless phonon becomes less probable owing to reduction of phonon number with small pulses during emission. Let's note also that at $T \rightarrow 0$ in this frequency range the damping becomes nonzero finite quantity due to the contribution of the processes with phonon emission.

The effect of phonon dispersion on exciton damping was also investigated for other crystals given in Table 2. It was revealed that in such crystals as $\mathrm{Cu}_{2} \mathrm{O}, \mathrm{GaP}, \mathrm{TlCl}$ the display of dispersion in damping is rather weak, because the damping itself for is a small quantity a wide interval of frequencies. So, for example, for $\mathrm{Cu}_{2} \mathrm{O}$ the damping does not exceed $1 \mathrm{~cm}^{-1}$ for the frequency range with phonon absorption, and $10 \mathrm{~cm}^{-1}$ for the range with phonon emission, respectively. We found out the greatest influence for $\mathrm{MgO}$ crystal. In this crystal at frequencies with phonon absorption the damping for maximal dispersion $(d=1)$ can exceed the damping in absence of dispersion more than twice. In this case, the damping in the frequency range with the phonon emission by almost two orders surpasses the damping in the range with phonon absorption.

The results of calculations of exciton damping for $\mathrm{AgBr}$ crystal are closes to illustrate above for $\mathrm{ZnS}$ crystal.

\section{Conclusions}

The dispersion of optical vibrations of the lattice influences on frequency dependence of exciton damping in ionic crystals. First of all, the frequency intervals with phonons absorption and emission change: with dispersion growth the frequency interval with absorption of an optical phonon extends, while the interval with the phonon emission is narrowed. It causes the reduction of intensity of damping of an exciton in the vicinity of the maximum of phonon absorption and its increase - in the region with maximum of phonon emission. Accordingly, on wings, the inverse dependence is found out: with growth of dispersion the value of damping with phonon absorp- tion enhanced, whereas with phonon emission it falls. Such frequency dependence is pronounced for crystals both with large and small exciton radii. However, for crystals with the large exciton radii it is more pronounced since the maximum of exciton-phonon coupling for them is displaced to the side of longer waves, owing to preference of exciton scattering on phonons with small wave vectors.

The most significant display of a dispersion in exciton damping for number of ionic crystals (Tab. 2) is revealed in $\mathrm{MgO}$ crystal, and is weakly pronounced for $\mathrm{Cu}_{2} \mathrm{O}$, $\mathrm{TlCl}$ and $\mathrm{GaP}$ crystals.

With increase of temperature, the damping of an exciton in the crystal gradually grows. This growth is approximately identical for majority of frequencies from one-phonon interval both in presence of dispersion and in its absence. However, for crystals with small exciton radii, there is a frequency interval, for which dependence of the damping on temperature behaves sharply in presence of the phonon dispersion. It results in phenomenon for which the damping in absence of the dispersion at low temperatures at first dominates on some frequency interval, and then, with increase of temperature, is compared and becomes even smaller than the damping in the presence of dispersion.

Depending on the energy interval the different behavior $\Gamma(T)$ is found out. So, in the range $\hbar \omega-E_{0} \leq \Omega_{0}$, $\Gamma \rightarrow 0$ at $T \rightarrow 0$, whereas in the range $\hbar \omega-E_{0} \geq \Omega_{0}$ it becomes finite quantity at zero temperatures owing to the not disappearing contribution of the processes with phonon emission.

\section{Acknowledgments}

The author expresses gratitude to Profs M.P. Lysytsja, M.Ya. Valakh and Dr A.M. Yaremko for helpful discussions of the work results.

\section{References}

1. J.A. Reissland, The Physics of Phonons. John Wiley \& Sons LTD, London, New York, Sidney, Toronto, (1973).

2. H.Mahan, Many-Partical Physics. Klumer Academic/Plenum Publishers, New-York, Boston, Dordrecht, London, Moscow (2000).

3. H. Ham and H. Spector // Phys. Rev. B, 62, pp. 13599-13603 (2000).

4. N.I. Grigorchuk // Phys. Rev. B, 55, pp. 888-893 (1997).

5. J.S. Weiner, P.S. Chemla, P.A.B. Miller, T.H. Wood, D. Sivco and A.Y. Cho // Appl. Phys. Lett., 46. pp.619-623 (1985).

6. R. Chen, D.L. Lin and B. Mendoza // Phys. Rev. B., 48 (16), pp. 11879-11882 (1993).

7. K.J. Nash and D.J. Mowbray // J. Lumin, 44, pp. 315-346 (1989).

8. M. Born and K.Huang, Dynamic theories of crystal lattices, Clarendon Press Oxford (1958), 488 p.

9. B.K. Ridley, Quantum processes in semiconductors, Clarendon Press, Oxford (1982), 304 p.

10. G. Dalling, A. Woods, Thermal Neutron Scattering. New York (1965).

11. M.J. Rice and E.J. Mele // Solid State. Commun. 35 (6), pp. 487-491 (1980); M.J. Rice, N.O. Lipari and S. Straussler // Phys. Rev. Lett., 39 (21), pp.1359-1362 (1977). 


\section{N.I. Grigorchuk: Influence of phonon dispersion on exciton damping in ionic crystals}

12. A.A. Gogolin // JETP, 92 (1), pp.152-164 (1987) (in Russian).

13. A.S. Alexandrov and J. Ranninger // Phys. Rev. B. 45 (22), pp.13109-13112 (1992).

14. N.I. Grigorchuk and I.Yu. Goliney // Phys. Rev. B, 60 pp. 5470-5475 (1999).

15. A.I. Ansel'm, Introduction into the theory of semiconductors. M.: Nauka (1978), 616 p. (in Russian).

16. B.K. Ridley // Phys. Rev. B. 49 (24), pp.17253-17258 (1994).

17. N.I. Grigorchuk // Ukr.Fiz.Zh., 23(3), pp.470-476 (1978) (in Ukrainian); Preprint of Institute of nuclear researches, No.13. 26 p (1976) (in Russian).
18. S.D. Mahanti and C.M. Varma // Phys.Rev. B., 6 (6), pp. 2209-2226 (1972).

19. G. Iadonisi and F. Bassani // Nuovo Cimento D., 2 (5), pp. $1541-1560$ (1983).

20. W. Yi, W. Kelin, W. Shaolong // Phys. Rev. B., 54 (3), pp. 1463 1466 (1966).

21. E.O. Kane // Phys. Rev. B., 18(12), pp. 6849-6855 (1978).

22. Acoustical crystals / Ed. by M.P. Shaskolskaja. M.: Nauka (1982), 632 p (in Russian).

23. H. Haken // Fortsch. Physik, 6 (6), pp. 271-334 (1958). 